\title{
Protection des données
} et LAA

\section{Réflexions sur les aspects juridiques de la protection des données en droit des assurances-accidents et en ce qui concerne plus particulièrement l'obligation de livrer des pièces}

\section{Fuchs ${ }^{a}$, U. Uttinger ${ }^{b}$, B. Soltermann ${ }^{c}$}

a Dr en droit, avocat, Suva

b lic. en droit, responsable de groupe de travail protection des données de l'ASA

c Dr méd, médecin-chef de l'Association Suisse d'Assurances ASA

Dans le Bulletin des médecins suisses du 15 août 2001, Hanspeter Kuhn, avocat, a largement commenté la question de la protection des données et celle $\mathrm{du}$ secret des patients en assurance-maladie [1]. Il apparaît indiqué, voire nécessaire, d'examiner ici la question de la protection des données du point de vue du droit de l'assurance-accidents (LAA), les auteurs se limitant toutefois à une présentation sommaire et pragmatique dans laquelle la remise des données et pièces médicales aux assureurs-accidents constitue l'élément essentiel.

\section{La situation}

Le $1^{\text {er }}$ janvier 2001, quelques modifications importantes de diverses lois d'assurance sociale - en relation avec la loi sur la protection des données - sont entrées en vigueur. Ce faisant, des lacunes ont été comblées et les lois ont été harmonisées. Presque parallèlement à cela, la Commission d'experts Geiser s'est, dans un rapport circonstancié, penchée sur la protection de la personnalité dans les assurancesmaladie et accidents sociales et privées et a présenté des propositions concrètes d'améliorations (voir aussi [1]). Celles-ci ne constituent toutefois, selon le rapport, que des «invitations à la réflexion à l'intention des autorités fédérales et des milieux concernés».

Le rapport d'experts analyse en détail les flux des données dans l'assurance de soins obligatoires selon LAMal et LAA (assureurs sociaux) ainsi que les condi-

\section{Correspondance:}

Lic. iur. Ursula Uttinger

Winterthur Versicherung

Hertistrasse 2c

Case postale 138

$\mathrm{CH}-8304$ Wallisellen

E-mail: ursula.uttinger@winterthur.com tions-cadre juridiques et la problématique de la protection des données dans les assurances maladie et accidents privées. Les différents assureurs qui, en partie, pratiquent même plusieurs types d'assurance présentent des besoins de données différents et sont soumis à des réglementations différentes en matière de protection des données. En raison de cette complexité, le rapport ne présente pas une solution standard mais propose des améliorations ponctuelles, pour la LAMal surtout, tandis que la LAA n'est concernée qu'à titre marginal. On voit déjà en cela qu'il y a de considérables différences entre la LAMal et la LAA.

\section{LAA et LAMal}

L'assurance-accidents obligatoire est une assurance spéciale liée à la cause (assurance de relation causale). L'assurance-maladie, en revanche, est et reste l'assurance de base pour toutes les atteintes à la santé. Les atteintes à la santé qui ne répondent pas aux conditions selon la LAA sont à la charge de l'assurancemaladie. Une constatation des circonstances de l'accident en ce qui concerne l'obligation de fournir la prestation, analogue à la maxime selon laquelle il appartient à l'assureur-accidents de rechercher les causes, n'est pas prévue dans l'assurance-maladie.

L'assurance-accidents obligatoire se fonde - à la différence de l'assurance-maladie - sur le principe de la prestation en nature. L'assureur doit fournir, à ses frais, les prestations de soins en nature. L'assureur se trouve être ainsi débiteur du médecin ou de l'hôpital. Les médecins et les hôpitaux, en tant que fournisseurs de prestations, agissent sur mandat de l'assureuraccidents sur base d'une relation juridique de droit public (cf. [2], p. 274 ss et 523 ss).

La maxime imputant la recherche de la cause à l'assureur-accidents fait aussi partie du principe de la prestation en nature. C'est-à-dire que l'assurance-accidents doit, de sa propre initiative et sans lien avec les allégations des parties intéressées, tirer d'office les faits et circonstances au clair. A relever à ce propos que l'obligation de fournir la prestation faite à l'assureur-accidents obligatoire présuppose un rapport de causalité naturel et adéquat entre l'accident et le dommage survenu. Dans la majorité des cas, ces élucidations s'effectuent au moyen de formules, l'employeur et/ou le médecin devant fournir des formules de déclaration standardisées (cf. art. 53 LAA). Dans le contexte du traitement administratif de l'accident, l'assureur-accident prend les dispositions nécessaires pour un traitement approprié et décide aussi du droit aux prestations et de leur montant (art. 47, 48 et 99 LAA). L'assuré ou ses survivants, mais aussi l'employeur et le médecin traitant, sont légalement obligés de participer à la constatation des circonstances. Cette obligation de collaboration s'étend naturellement aussi aux données personnelles (voir à ce sujet les art. 47 et 54 a LAA ainsi que les art. 53, 55 et 56 OLAA). 
Dans l'assurance-accidents selon la LAA, la question de la causalité joue un rôle important et présente des aspects tant médicaux que juridiques. Cela veut dire que la causalité ne peut être normalement appréciée qu'en commun par l'administration et le service médical. Il faut enfin mentionner la large obligation de collaborer de l'assuré (art. 55 OLAA). Afin d'être en mesure de satisfaire aux exigences spécifiques de la LAA (appréciation de questions concernant l'accident/la récidive, les maladies professionnelles, les questions de causalité en général, les capacités de travail, l'appréciation de l'exigibilité), la SUVA a mis sur pied sa propre section médicale et gère en outre un système de médecins d'arrondissement. Ces médecins d'arrondissement sont des employés de la SUVA dans les agences et travaillent étroitement avec l'administration. Leurs tâches se règlent sur la LAA. Les compagnies d'assurances privées qui opèrent dans le secteur LAA confient en revanche leurs mandats de conseil à des médecins indépendants ou à des médecins d'hôpitaux.

\section{Art. 54 a LAA et son interprétation ou: quelles données les hôpitaux et les médecins doivent-ils remettre aux assureurs-accidents?}

Evolution depuis l'entrée en vigueur de la loi fédérale sur la protection des données (LPD) Depuis l'entrée en vigueur de la LPD, la remise de rapports médicaux à l'administration a suscité, à maintes reprises, des discussions entre assureurs-accidents (privés comme SUVA aussi) et les divers fournisseurs de prestations, du fait notamment que le Préposé fédéral à la protection des données (PFPD) prônait la réserve. Si les assureurs-accidents suivaient l'avis jusqu'ici émis par le PFPD et quelques hôpitaux, il ne serait guère possible de mettre en pratique l'assurance-accidents conformément à la loi (notamment l'appréciation de la causalité et des prestations). Le principe de la prestation en nature se trouverait massivement affecté. La question de savoir comment il faut apprécier le nouvel art. 54 a LAA sous cet éclairage revêt de ce fait une grande importance.

\section{Le nouvel art. 54 a LAA}

Ce nouvel article, en vigueur depuis le $1^{\text {er }}$ janvier 2001, stipule que les fournisseurs de prestations (soit les médecins et les hôpitaux) sont, par analogie, tenus de transmettre aux assureurs toutes les indications nécessaires pour qu'ils puissent se prononcer sur le droit à des prestations. Dans un article récemment paru, Roger Peter, avocat et juge suppléant au Zürcher Sozialversicherungsgericht, se livre à des réflexions approfondies sur ce sujet («Besteht eine Pflicht des Arztes auf Herausgabe von Daten seines Patienten an das Durchführungsorgan der obligatorischen Unfallversicherung?» Soit, en résumé: le médecin est-il autorisé à révéler des données concernant son patient à l'organe d'exécution de l'assurance-accidents chargé de l'établissement des faits et, le cas échéant, quand, sous quelle forme et dans quelle mesure?) [3]. Compte tenu du PFPD ainsi que de l'évolution de la LAA, on en arrive, pour l'essentiel, aux conclusions suivantes:

- Ce ne sont pas seulement les fournisseurs effectifs de prestations LAA qui, suite à la survenance de l'événement assuré, tombent sous le coup de l'art. 54 a LAA, mais aussi toutes les personnes exerçant une activité dans le domaine médical qui pourraient contribuer à faire de la lumière sur les circonstances permettant de fonder un droit.

- Selon l'art. 47 LAA en relation avec l'art. 55 OLAA, la personne assurée doit donner à l'organe d'exécution tous les renseignements et pièces nécessaires (par ex. rapports médicaux, rapports d'expertise, radiographies) dont celui-ci a besoin pour déterminer les circonstances et les suites de l'accident et pour fixer les prestations d'assurance. La personne assurée doit, en outre, autoriser les médecins traitants à fournir de tels documents et à donner des renseignements. Cette interprétation historique correspond à l'objectif de l'art. 54 a. En d'autres termes, on peut induire de l'art. 54 a (seconde phrase), une injonction implicite allant dans le sens que l'organe d'exécution de l'assurance-accidents obligatoire peut, pour établir les circonstances permettant de fonder un droit, exiger du personnel exerçant une activité dans le domaine médical, non seulement des informations, mais en principe aussi des documents. Par ceuxci, il faut entendre des rapports médicaux, des expertises, des "prints", des radiographies, etc.

- Un médecin ou un hôpital ne peut refuser de remettre de telles pièces que dans trois cas d'exception, à savoir:

- si, pour des raisons personnelles, le fait de répondre aux questions ou de remettre des documents entraînerait un préjudice pour le médecin et/ou ses proches parents;

- s'il s'agit de données personnelles exclusivement destinées à un usage personnel (par ex. notes personnelles sur la feuille de maladie de l'assuré, remarques de type organisationnel);

- si et pour autant qu'il s'agisse de données sans importance et sans rapport avec le cas. Ainsi, par exemple, l'organe d'exécution ne doit pas avoir connaissance d'un traitement psychiatrique antérieur pour apprécier le traitement curatif d'une simple lésion du ménisque. Les circonstances permettant de fonder un droit doivent toutefois être interprétées plutôt largement (cf. la définition dans [3], p. 149).

La question de l'importance légale

Lorsqu'il s'agit de l'importance légale, soit de la question de savoir quelles sont les données qui doivent être considérées comme "indispensables" ou "nécessaires", il existe une certaine marge d'appréciation (voir sous "LAA et LAMal» ci-dessus). Il faut alors partir des circonstances permettant de fonder un droit, soit des faits qui ont une influence déterminante sur la prétention à apprécier. Dans des cas plus complexes, un simple règlement par le biais de formules à remplir ne suffit pas et l'organe d'exécution doit, 
par exemple, voir si un événement s'est réellement produit, s'il peut être qualifié d'accident ou si, éventuellement, il s'agit d'une lésion corporelle assimilée, s'il y a des antécédents et lesquels, s'il y a une relation causale, etc. Afin de ne pas se voir plus tard reprocher par le tribunal que les circonstances n'ont pas été suffisamment élucidées, en ces cas l'assureuraccidents doit se procurer des moyens de preuve complets.

Obligation du secret professionnel

Il ne faut pas perdre de vue, dans ce contexte, que la LAA porte aussi une disposition concernant le secret médical auquel toutes les personnes pratiquant la LAA sont soumises (voir art. 102 LAA). Celles-ci doivent, à l'égard des tiers, garder le secret sur les données et les informations dont elles ont eu connaissance du fait de leur activité. Qui contrevient à cette obligation du secret professionnel est passible d'un emprisonnement allant jusqu'à six mois ou d'une amende (art. 112 LAA).

\section{En conclusion}

La LAMal et la LAA présentent de sensibles différences. Pour la LAA, le principe de la prestation en nature et celui de la maxime selon laquelle il appartient à l'assureur-accidents de déterminer les causes (y compris l'appréciation du lien de causalité) sont déterminants. Ceci fait que le secret médical et la protection des données ne peuvent, en matière d'assurance-accidents, être traités de la même manière (restrictive) qu'en assurance-maladie. Davantage d'informations sont nécessaires et licites en matière de LAA.
Dans le contexte de l'obligation de remettre des pièces et indications prévue à l'art. 54 a LAA, il n'y a pas de secret médical et le dépositaire du secret (patient ou tiers) n'a pas non plus l'obligation de donner au médecin traitant une autorisation formelle, tacite ou supposée, pour révéler le secret.

Si le médecin ou l'hôpital refuse l'information ou la production de pièces raisonnablement exigibles, une exécution forcée ou une amende immédiate ne peuvent être ordonnées vu l'absence de base légale. A défaut de preuves documentées juridiquement suffisantes, l'assureur devra faire observer à l'hôpital ou au médecin que la prétention à la prestation devra être refusée si les pièces ne sont pas fournies dans les délais, l'assuré se trouvant finalement être la victime de cette situation.

\section{Références}

1 Kuhn HP. Protection des données et LAMal. Réflexions concernant la sphère privée, le secret médical et la protection des données dans l'assurance sociale à l'exemple de la LAMal. Bull Med Suisse 2001;82(33):1707-17.

2 Maurer A. Schweizerisches Unfallversicherungsrecht. Bern: Stämpfli; 1985.

3 Peter R. Besteht eine Pflicht des Arztes auf Herausgabe von Daten seines Patienten an das Durchführungsorgan der obligatorischen Unfallversicherung? Schweiz Z Sozialversicherung 2001 ;45:147-62. 